\title{
Surface-state engineering for interconnects on H-passivated $\mathrm{Si}(100)$
}

\author{
Mikaël Kepenekian, ${ }^{*}{ }^{\dagger}$ Roberto Robles, ${ }^{\dagger}$ Christian Joachim, ${ }^{\ddagger}$ and Nicolás Lorente ${ }^{\dagger}$ \\ Centro de Investigación en Nanociencia y Nanotecnología CIN2 (CSIC-ICN), Campus de la UAB, \\ E-08193 Bellaterra, Spain, GNS \& MANA satellite, Centre d'Elaboration des Matériaux et \\ d'Etudes Structurales (CEMES), CNRS, 29 rue J. Marvig, 31055 Toulouse Cedex, France, and \\ Institute of Materials Research and Engineering, 3 Research Link, Singapore 117602, Singapore \\ E-mail: mikael.kepenekian@cin2.es
}

\begin{abstract}
Surface-state engineering strategies for atomic-size interconnects on $\mathrm{H}$-passivated $\mathrm{Si}(100)$ surfaces are explored. The well-known simple interconnect formed by removing H-atoms from one of the $\mathrm{Si}$ atoms per dimer of a dimer row along the $\mathrm{Si}(100)$ surface is poorly conducting. This is because one-dimensional-like instabilities open electronic gaps. Here, we explore two strategies to reduce the instabilities: spacing the dangling bonds with $\mathrm{H}$ atoms and changing the geometry by increasing the lateral size of the wires. The resulting wires are evaluated using density functional theory. Surprisingly, zig-zag dangling-bond wires attain atomically-confined conduction properties comparable with the conduction of free-standing metallic monoatomic wires. These results hint at band-engineering strategies for the development of electronically driven nanocircuits.
\end{abstract}

Keywords: silicon interconnects; nanowires; electron transport; DFT

\footnotetext{
${ }^{*}$ To whom correspondence should be addressed

CIN2

FEMES

"IIMRE
} 
The development of molecular electronics strongly relies in our ability to individually connect single atoms and molecules. ${ }^{1}$ A quantum leap was effected when the manipulation of individual atoms and molecules became possible. This has permitted to connect atomic-scale devices between two electrodes fabricated in a metallic junction via mechanical, ${ }^{2}$ electromigration ${ }^{3}$ and scanningprobes techniques. ${ }^{4-6}$ The presence of a supporting surface is very important in these approaches with silicon surfaces playing a leading role due to their physicochemical properties as well as the existing wealth of silicon-based techniques. ${ }^{7-12}$ Unfortunately, the development of working devices is being deterred by the lack of low-resistivity leads and interconnects of the same size scale as the active atomic device.

Promising breakthroughs in silicon-based interconnects have been the creation of many types of silicon nanowires. ${ }^{13,14}$ However, Björk and co-workers ${ }^{15}$ have shown that the resistivity of doped wires increases exponentially as the wire's diameter decreases below $\sim 10 \mathrm{~nm}$. As a remedy for this limitation, Weber et al. ${ }^{16}$ have recently embedded nanowires on a silicon surface by selectively doping using scanning-probe technology. Previously, an alternative but related approach has been proposed $^{17-19}$ in which surface electronic states were engineered, taking advantage of the very local nature of silicon bonds. By creating dangling-bond nanowires on silicon surfaces, surface electronic states can be precisely tuned. This has been shown by extended Hückel calculations ${ }^{20}$ of different dangling-bond structures including wires formed by a single and a double row of dangling bonds on a H-passivated Si (100) surface. Surface electronic states are very interesting as interconnects since their current leakage is strictly zero. Indeed, an ideal requirement for surface interconnects is that all of the current remain on the surface hence avoiding electron leaks from the surface to the bulk. Such a phenomenon is characterized by a bulk contribution to the electronic transmission. The enhanced atom manipulation ability of scanning probes have permitted the creation of quasi 1-D surface states with extraordinary properties. ${ }^{18,19,21-26}$ Experimental $^{18}$ and theoretical studies ${ }^{17,21,22,24,25,27}$ have shown that in the limit of one-atom wide dangling-bond wires, 1-D correlations are very strong and instabilities set in creating electronic gaps in the wire's electronic structure. As recently shown, this has detrimental consequences in the wires' electron- 
transport properties, ${ }^{28}$ reducing the possibilities of dangling-bond wires as alternative interconnects to free-standing ${ }^{13,14}$ or embedded ${ }^{16}$ nanowires. Nevertheless, the richness of surface-related techniques should allow us to create surface band-engineering strategies that go beyond the 1-D limitations.

In this Letter, we show how to improve on the poor transport properties of dangling-bond nanowires that were revealed in Ref. ${ }^{28}$ In order to achieve this, different arrangements of the nanowires are explored since the cause of the above large correlations can be traced back to the confinement of the electronic structure of dangling bonds. Hence, arrangements that allow for more extended electronic states, should in principle reduce electronic correlations and generally improve charge transport properties. Here, we study three arrangements and we compare them with the results of the strongly correlated case of a single-row dangling-bond wire, Figure 1 (a). The first attempt is to increase the charge delocalization by increasing the inter dangling-bond distance. Here the hope is that hybridizations are still large enough while reducing the electronic Coulomb repulsion. The corresponding half-row wire is shown in Figure 1 (b). Another arrangement is shown in Figure 1 (c), the zig-zag wire that is a mid-case between the previous single-row and half-row wires. Finally, Figure 1 (d) studies the effect of increasing the density of dangling-bond states to reduce electron localization. As expected correlations effects are reduced due to the larger extension of the electronic structure and the charge transport properties of the zig-zag and doublerow wires are very much improved. Our study concludes that, in particular, zig-zag nanowires can be an interesting alternative for surface interconnects.

First-principles calculations are based on density functional theory (DFT) as implemented in SIESTA. ${ }^{29,30}$ Calculations have been carried out with the GGA functional in the PBE form, ${ }^{31}$ Troullier-Martins pseudopotentials, ${ }^{32}$ and a double- $\zeta$ polarized basis set of finite-range numerical pseudoatomic orbitals for the valence wave functions. ${ }^{33}$ Surfaces were modeled using a slab geometry with eight silicon layers and a $2 \times 2$ surface unit cell. The electronic structure was converged using a $5 \times 3 \times 1 k$-point sampling of the Brillouin zone. The geometrical relaxation has been performed until forces on the upper four layers were smaller than $0.04 \mathrm{eV} / \AA$. In the present 

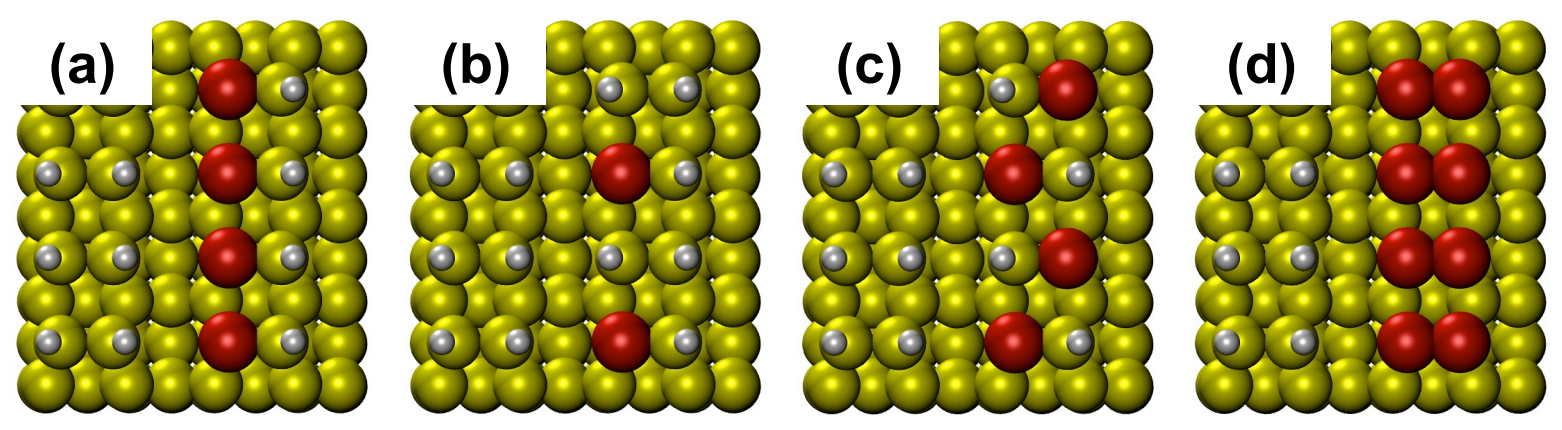

Figure 1: Atomic structures of (a) the single-row, (b) the half-row, (c) the zig-zag, and (d) the double-row dangling-bond (dangling-bond) wires. $\mathrm{H}$ atoms are depicted in white, $\mathrm{Si}$ atoms in yellow and red when holding a dangling-bond.

work, we deal with infinite wires hence, the electron transmission is strictly fixed by the number of contributing electronic bands at a given electron energy. For a fast, qualitative evaluation of the conducting quality of the considered wires, we evaluate an indicative electron current by using the Landauer-Büttiker current expression. ${ }^{34}$

The transmission of the AFM wire, Figure 2 (a), differs from the previously published ones ${ }^{28}$ because the present wire is infinite. As a consequence, the sharp structures due to the geometrical confinement of the finite wires ${ }^{28}$ develop into broader bands. The two plateaus at a transmissionper-spin equal to one are purely due to electronic states confined to the wires. Let us notice that the AFM solution brings no discrimination between spins. Therefore no spintronics properties such as spin selective transport ${ }^{35}$ are expected from the single-row wire. Figure 2 (b) shows one of the electronic states just at the onset of the empty-state transmission plateau. While the main amplitude of the state is located at the dangling-bond sites, there is an exponential decrease of the amplitude as we move away from the wire into the bulk. This is indeed a dangling-bond surface state. At $\sim+0.6 \mathrm{eV}$ and $\sim-0.8 \mathrm{eV}$ transport through the conduction and valence bands starts. In the absence of doping, the chemical potential is in the gap so that, in a simple picture of the possible electron current that the wire can sustain, the bias needs to be ramped up to $\sim 0.5 \mathrm{~V}$ to find a non-zero current, Figure 3 (black line). Despite the poor conducting properties of the AFM infinite wire, there is a finite bias window (between $\sim 0.5$ and $\sim 1.2 \mathrm{~V}$ ) where conduction is strictly performed by the engineered surface state. For biases larger than $1.2 \mathrm{~V}$ the conduction-band states 

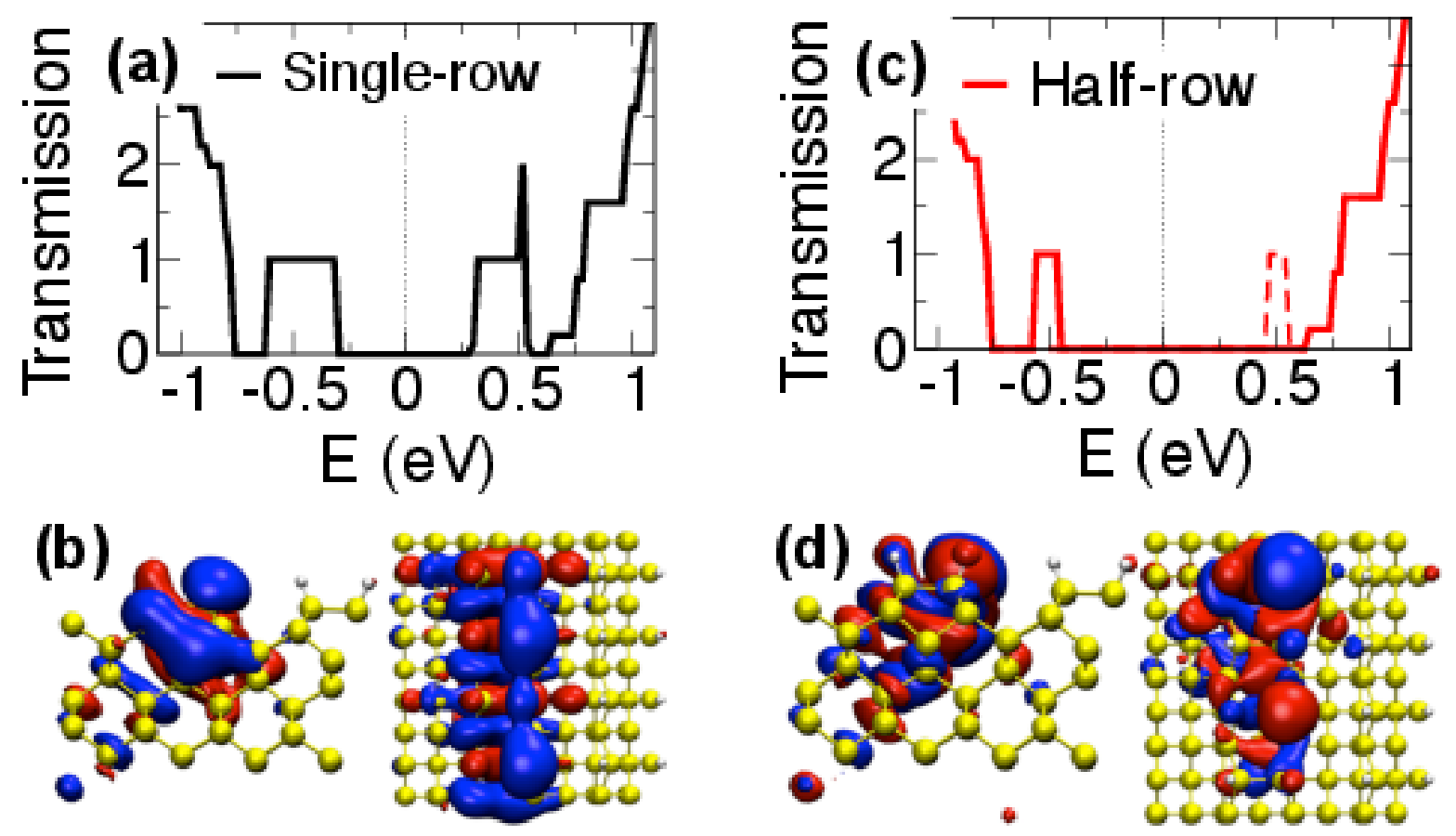

Figure 2: (a) and (c) Transmission function with respect to electron energy for the AFM singlerow and the half-row dangling-bond wires, respectively. The chemical potential is taken as the origin of energies. (b) and (d) Side and top view of the surface Bloch wavefunction of empty states for the AFM and half-row wires, respectively. Red and blue stand for the positive and negative wavefunction amplitude values.

start contributing and the current is not confined anymore.

The half-row wire is an attempt to modify the surface electronic structure by creating a KronigPenney structure that will modulate the surface band. Indeed, the alternated passivation of danglingbonds along the wire becomes a periodic confining potential. This strategy has been used in the modification of the $\mathrm{Au}(111)$ surface state by one-dimensional arrays of adsorbed molecules. ${ }^{36}$ Surprisingly, the periodic potential does not induce states in the AFM gap, but it leads to a FM ground state with the consequence of enhancing the electronic gap, Figure 2 (c). The Kronig-Penney-like potential imposes a confinement that sharpens the previous transmission plateaus with the novelty that the occupied plateau corresponds to majority spin carriers and the empty one to the minority spin in a broken symmetry description of electron transport. Both plateaus are symmetrical with respect to the Fermi energy. Therefore, by applying a bias, one cannot trigger the transmission of majority spins without getting the same contribution from minority ones. Hence, only in the 


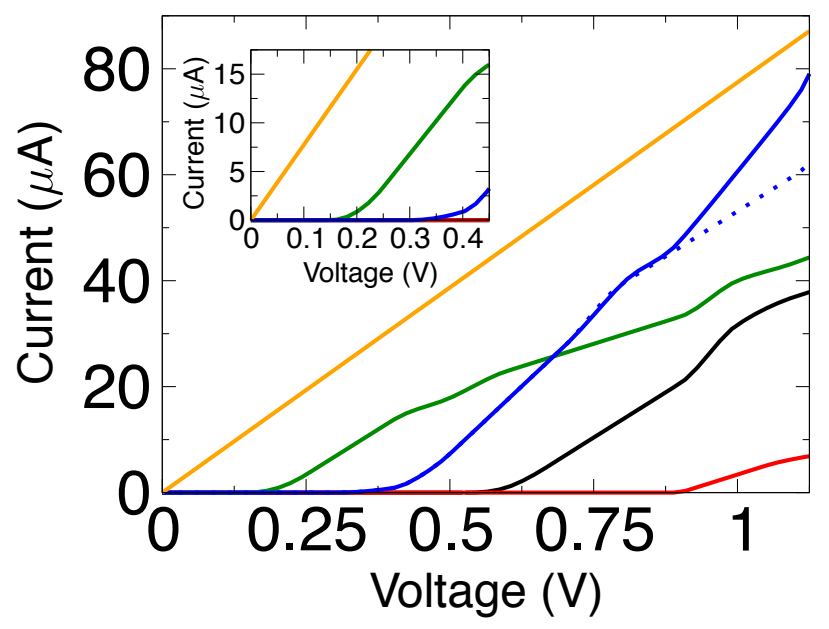

Figure 3: I-V curves of the single-row AFM (black), half-row (red), zig-zag (green) and doublerow (blue) dangling-bond wires. In this last case, a sizeable bulk contribution to the current starts in the presented bias window. In dashed lines, the surface-state contribution of the double-row wire is shown. In full line, the full current is presented. The yellow line is the I-V of a one-channel metallic wire, such as a free-standing monoatomic gold wire.

presence of doping and some symmetry-breaking effect, we could expect to enhance the transmission of one spin and use the half-row wire as a spin filter. The transport electronic states, Figure 2 (d), show a similar degree of surface confinement as for the AFM wire while presenting a significant amplitude reduction over the passivating $\mathrm{H}$-atoms. The FM solution and Kronig-Penney confinement leads to an important reduction of surface driven current, Figure 3 (red line).

The natural extension of the single-row wire studies is to reduce the correlation effects causing the AFM and FM solutions by increasing the lateral dimension of the engineered surface states. We first displace one of the passivating $\mathrm{H}$ atoms of the AFM wire in order to create a zig-zag dangling-bond structure that, while maintaining part of the 1-D character, reduces correlations by decreasing confinement. Our ground state studies yield that electronic correlations get substantially reduced and the solution becomes not magnetic. However, an important buckling of the surface dimers is found, exceeding the clean surface dimer buckling by $\sim 0.1 \AA$ in the vertical dimer displacement, as shown in Figure 4 (a). The dimer distortion leads to a Peierls-like opening of a gap in the dangling-bond surface state. However, our objective has been partially achieved since the electronic gap has considerably decreased. It is now $\sim 0.2 \mathrm{eV}$ against $\sim 0.7 \mathrm{eV}$ for the AFM 
(a)

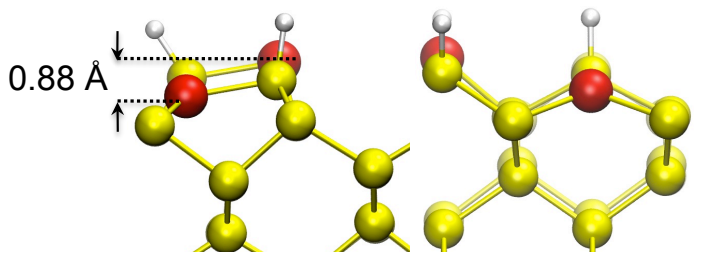

(b)

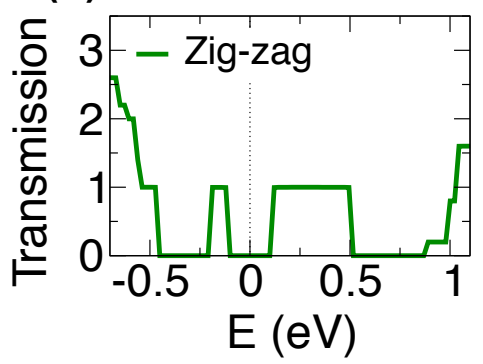

(c)

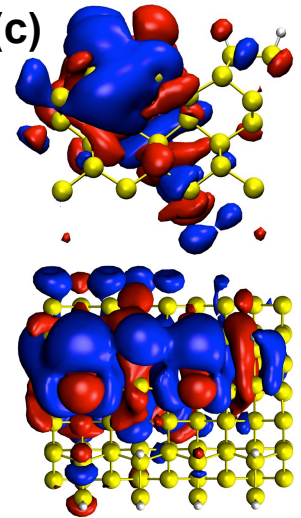

Figure 4: (a) Ground-state geometry of the zig-zag dangling-bond wire. $\mathrm{H}$ atoms are shown as white, Si atoms are depicted as yellow and as red when holding a dangling-bond. (b) Transmission function with respect to electron energy. The chemical potential is taken as the origin of energies. (c) Side and top view of an empty-state Bloch wavefunction $(E=0.25 \mathrm{eV})$ of the wire. Red and blue stand for the positive and negative wavefunction amplitude values.

wire and $\sim 0.9 \mathrm{eV}$ for the half-row one. The H-diffusion barriers are within $1 \mathrm{eV}$ for different diffusion paths along dangling bonds. ${ }^{27}$ Hence, even though the zig-zag wire is $110 \mathrm{meV} / \mathrm{dimer}$ higher in energy than the single-row wire, the proposed structure should be achievable with present scanning-probe manipulation means.

Interestingly, the transmission function of the zig-zag wire, Figure 4 (b), shows a 0.4-V wide plateau of 1 conduction channel. This channel corresponds to a surface state as can be seen in one of the wavefunctions corresponding to the energy $0.25 \mathrm{eV}$, Figure 4 (c). The effect of the improved transmission can be seen in Figure 3 (green line). Indeed, the zig-zag wire is capable of holding the largest current below the onset of bulk conduction among the four wires explored here. The extended 1-channel transmission plateau permits us to claim that the conduction properties should be comparable to those of good conductors such as free standing monoatomic gold wires. ${ }^{37}$ For comparison, Figure 3 shows the I-V of an ideal one-channel metallic wire (such as a the monoatomic gold wire). In a medium-range bias, about $0.5 \mathrm{~V}$, the zig-zag wire carries only half the current of a metallic wire.

Leakage currents due to bulk conduction band states appear for electron energies above 0.75 $\mathrm{eV}$. When the bias is large enough to include bulk states, part of the electron transport will proceed 
through theses states and will be lost into the bulk material. Hence, our calculations predict that zigzag wires can be very good interconnects with large surface-confined currents within a comfortable bias window below $1 \mathrm{~V}$.

Finally, a wire formed by a row of dangling-bond dimers, Figure 1 (d), presents the smallest charge confinement of the shown wires and hence a more metallic-like electronic structure. As a consequence, electron correlation is the lowest, which makes magnetic solutions very high in energy. And indeed, the double-row wire spin-polarized solution is $290 \mathrm{meV} / \mathrm{dimer}$ higher in energy than the non-magnetic solution. The distorted system adopts a buckled geometry similar to the case of the non-passivated $\mathrm{Si}(100)$ surface, Figure 5 (a). In the buckled configuration, the two Si atoms of one dimer move $0.79 \AA$ in the vertical direction. In addition, the subsurface atoms undergo a slight dimerization of $0.24 \AA$ along the wire's direction. As for the $\operatorname{Si}(100)$ surface case, the $\pi$-backbond is perturbed by the distortion and a gap opens in the electronic structure. The consequence in the transmission, Figure 5 (b), is again two surface-state transmission bands, one for occupied and the other one for empty states, similar to the zig-zag case. However, here, the transmission at $-0.4 \mathrm{~V}$ contains valence band channels. Hence, from $0.8 \mathrm{~V}$ on, the current is composed of surface-state and bulk-state contributions which is against the good behavior of a surface interconnect. Figure 3 (blue line) shows the estimated surface contribution in dashed lines and the total current in full line. Beyond $0.8 \mathrm{~V}$, there is an increasing bulk contribution to the current. The bulk contribution rapidly adds conduction channels that yield a faster-growing I-V curve as compared with the ideal metallic wire (yellow). Hence, despite of presenting a sizeable surface current, the present wire would be a worse surface interconnect than the zig-zag because of its larger band gap and an increasing leakage current above $0.8 \mathrm{~V}$.

In summary, one-dimensional atomic-size interconnects are prone to instabilities that open electronic gaps due to strong correlations. The effect on the quality of electron transport in the interconnect is devastating, greatly reducing the currents that can be sustained by the interconnects. We have shown this in two types of one-dimensional-like wires formed by surface states on a H-passivated $\mathrm{Si}(100)$ surface. The first type of wire corresponds to removing a single line 
(a)

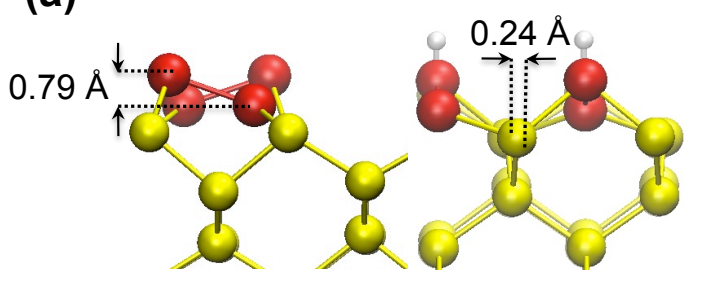

(b)

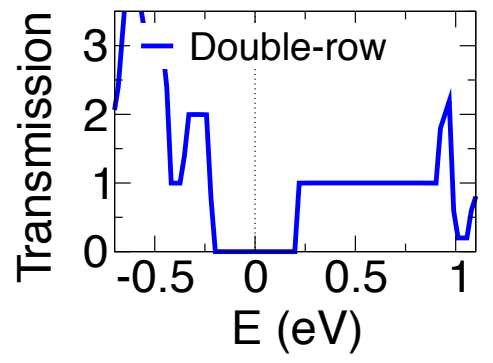

(c)

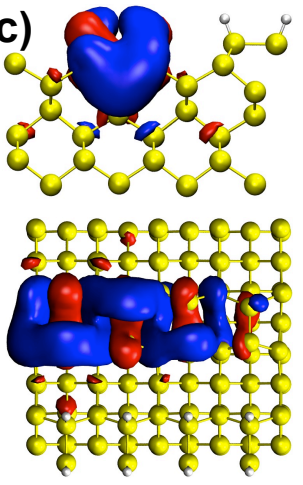

Figure 5: (a) Relaxed geometry of the ground state of the double-row dangling-bond wire. H atoms are shown as white, $\mathrm{Si}$ atoms are depicted as yellow and as red when holding a dangling-bond. (b) Transmission function with respect to electron energy. The chemical potential is taken as the origin of energies. (c) Side and top view of an empty-state Bloch wavefunction $(E=0.25 \mathrm{eV})$ of the wire. Red and blue stand for the positive and negative wavefunction amplitude values.

of $\mathrm{H}$ atoms along a dimer row of the $\mathrm{Si}(100)$ surface. Electronic correlations force the ground state to be antiferromagnetically coupled and to an important electronic gap. Two strategies are then undertaken to reduce the electronic gap. The first one is to add a periodic potential in the form of alternated $\mathrm{H}$ atoms with the single-row dangling bonds. However, electronic correlations are still strong and the ground state is ferromagnetically coupled. The other strategy is to reduce the one-dimensional confinement by increasing the wire dimensions. Two wires are explored: a zig-zag wire, where the dangling-bond alternates sites along the dimer row and a double-row wire where both sites are dangling bonds. In both cases the electronic correlations are sufficiently reduced to have a non-magnetic ground state. As a consequence the electronic band gaps diminish and the transport properties improve. However, valence-band states start contributing to the transmission, implying that a fraction of the electron current will be lost into the bulk material. The corresponding bulk contribution to the electron current is larger and takes place at lower bias for the double-row wire. Hence, the zig-zag wire constitutes the best solution to increasing electron transport in surface-state engineered interconnects.

This study reveals that surface-state based interconnects on Si surfaces can be tuned to decrease the correlations induced by the one-dimensionality of atomic-size interconnects. The suggested strategy consists in using a combination of geometry properties that reduce confinement but main- 
tains a reduced coupling with bulk states. We have achieved this by proposing a zig-zag geometry of dangling-bond wire that yields surface driven currents within a factor of two at $0.5 \mathrm{~V}$ of the current in a free-standing monoatomic gold wires. This work presents strategies for the development of interconnects among nanodevices that use electron currents.

\section{Acknowledgement}

The authors acknowledge financial support from the European-Union Integrated Project AtMol (http://www.atmol.eu).

\section{References}

(1) Joachim, C.; Gimzewski, J. K.; Aviram, A. Nature 2000, 408, 541-548.

(2) J.M. van Ruitenbeek, J.; Alvarez, A.; Pineyro, I.; Grahmann, C.; Joyez, P.; Devoret, M.; Esteve, D.; Urbina, C. Rev. Sci. Instrum. 1996, 67, 108.

(3) Park, H.; Lim, A.; Alivisatos, A.; Park, J.; Euen, P. Appl. Phys. Lett. 1999, 75, 301.

(4) Eigler, D. M.; Schweizer, E. K. Nature 1990, 344, 524.

(5) Lopinski, G. P.; Wayner, D. D. M.; Wolkow, R. A. Nature 2000, 406, 48.

(6) Dappe, Y. J.; González, C.; Bulou, H.; Berndt, R. Nano Lett. 2011, 11, 3142 - 3146.

(7) Kane, B. E. Nature 1998, 393, 133.

(8) Gimzewski, J. K.; Joachim, C. Science 1999, 283, 1783.

(9) Hallam, T.; Reusch, T. C. G.; Oberbeck, L.; Curson, N. J.; Simmons, M. Y. J. Appl. Phys. 2007, 101, 034305.

(10) Fuhrer, A.; Fuechsle, M.; Reusch, T. C. G.; Weber, B.; Simmons, M. Y. Nano Lett. 2009, 9, 707.

(11) Fuechsle, M.; Mahapatra, S.; Zwanenburg, F. A.; Friesen, M.; Eriksson, M. A.; Simmons, M. Y. Nature Nanotech. 2010, 5, 502. 
(12) Fuechsle, M.; Miwa, J. A.; Mahapatra, S.; Ryu, H.; Lee, S.; Warschkow, O.; Hollenberg, L. C. L.; Klimeck, G.; Simmons, M. Y. Nature Nanotech. 2012, 7, 242.

(13) Cui, Y.; Lieber, C. M. Science 2001, 291, 851-853.

(14) Rurali, R. Rev. Mod. Phys. 2010, 82, 427-449.

(15) Björk, M. T.; Schmid, H.; Knoch, J.; Riel, H.; Riess, W. Nature Nanotechnology 2009, 4, 103.

(16) Weber, B.; Mahapatra, S.; Ryu, H.; Lee, S.; Fuhrer, A.; Reusch, T. C. G.; Thompson, D. L.; Tee, W. C. T.; Klimeck, G.; Hollenberg, L. C. L.; Simmons, M. Y. Science 2012, 335, 64.

(17) Watanabe, S.; Ono, Y. A.; Hashizume, T.; Wada, Y. Phys. Rev. B 1996, 54, R17308.

(18) Hitosugi, T.; Heike, S.; Onogi, T.; Hashizume, T.; Watanabe, S.; Li, Z.-Q.; Ohno, K.; Kawazoe, Y.; Hasegawa, T.; Kitazawa, K. Phys. Rev. Lett. 1999, 82, 4034.

(19) Doumergue, P.; Pizzagalli, L.; Joachim, C.; Altibelli, A.; Baratoff, A. Phys. Rev. B 1999, 59, 1591015916.

(20) Raza, H. Phys. Rev. B 2007, 76, 045308.

(21) Cho, J.-H.; Kleinman, L. Phys. Rev. B 2002, 66, 235405.

(22) Bird, C. F.; Bowler, D. R. Surf. Sci. 2003, 531, L351-L355.

(23) Soukiassian, L.; Mayne, A. J.; Carbone, M.; Dujardin, G. Surf. Sci. 2003, 528, 121.

(24) Lee, J. Y.; Cho, J.-H.; Zhang, Z. Phys. Rev. B 2009, 80, 155329.

(25) Lee, J.-H.; Cho, J.-H. Surf. Sci. 2011, 605, L13-L15.

(26) Bianco, F.; Owen, J. H. G.; Köster, S. A.; Mazur, D.; Renner, C.; Bowler, D. R. Phys. Rev. B 2011, 84, 035328 .

(27) Robles, R.; Kepenekian, M.; Monturet, S.; Joachim, C.; Lorente, N. J. Phys.: Condens. Matter 2012, 24, 445004. 
(28) Kepenekian, M.; Novaes, F. D.; Robles, R.; Monturet, S.; Kawai, H.; Joachim, C.; Lorente, N. J. Phys.: Condens. Matter 2013, 25, 025503.

(29) Soler, J. M.; Artacho, E.; Gale, J. D.; García, A.; Junquera, J.; Ordejón, P.; Sánchez-Portal, D. J. Phys.: Condens. Matter 2002, 14, 2745-2779.

(30) Artacho, E.; Anglada, E.; Diéguez, O.; Gale, J. D.; García, A.; Junquera, J.; Martin, R. M.; Ordejón, P.; Pruneda, J. M.; Sánchez-Portal, D.; Soler, J. M. J. Phys.: Condens. Matter 2008, 20, 064208.

(31) Perdew, J. P.; Burke, K.; Ernzerhof, M. Phys. Rev. Lett. 1996, 77, 3865.

(32) Troullier, N.; Martins, J. L. Phys. Rev. B 1991, 43, 1993.

(33) Artacho, E.; Sánchez-Portal, D.; Ordejón, P.; García, A.; Soler, J. M. phys. stat. sol. (b) 1999, 215, 809.

(34) Datta, S. Electronic Transport in Mesoscopic Systems; Cambridge University Press, 2007.

(35) Jansen, R. Nature Mater. 2012, 11, 400.

(36) Gonzalez-Lakunza, N.; Fernández-Torrente, I.; Franke, K. J.; Lorente, N.; Arnau, A.; Pascual, J. I. Phys. Rev. Lett. 2008, 100, 156805.

(37) Pascual, J. I.; Méndez, J.; Gómez-Herrero, J.; Baró, A. M.; García, N.; Binh, V. T. Phys. Rev. Lett. 1993, 71, 1852-1855. 


\section{Table of Contents Graphic}

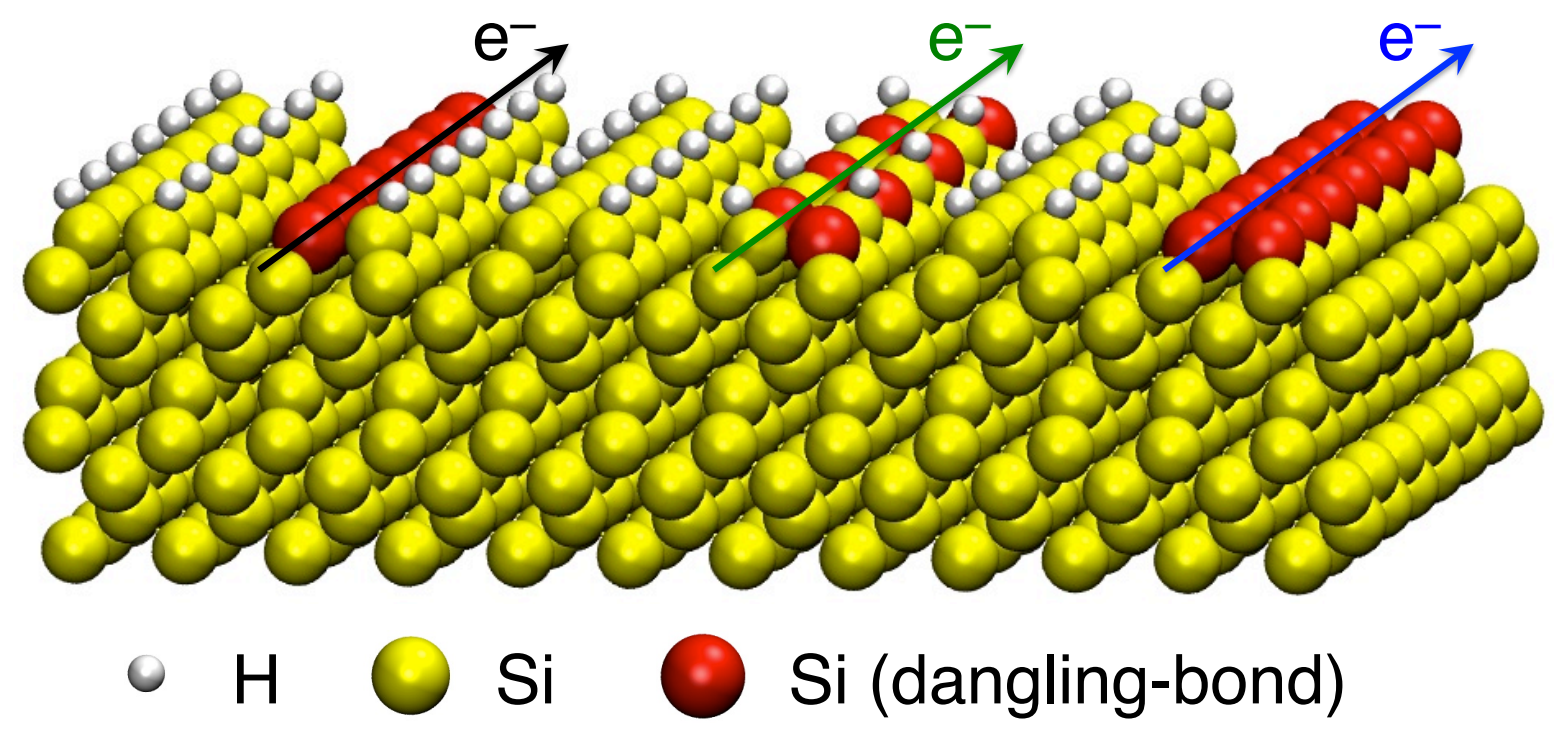

